\title{
Spitzer reveals what's behind Orion's Bar
}

\author{
Robert H. Rubin ${ }^{1,2,3}$ \\ ${ }^{1}$ NASA Ames Research Center, M.S. 245-6, Moffett Field, CA 94035-1000, USA \\ email: rubin@cygnus.arc.nasa.gov \\ ${ }^{2}$ Orion Enterprises \\ ${ }^{3}$ Kavli Institute for Astronomy \& Astrophysics, Peking University
}

\begin{abstract}
We present Spitzer Space Telescope observations of 11 regions in the Orion Nebula all southeast of the Bright Bar. Our Cycle 5 program obtained deep spectra with both the IRS short-high ( $\mathrm{SH}$ ) and long-high ( $\mathrm{LH})$ modules with aperture grid patterns chosen to very closely match the same area in the nebula. Previous IR missions observed only the inner few arcmin (the 'Huygens' region). The extreme sensitivity of Spitzer in the 10-37 $\mu \mathrm{m}$ spectral range permitted us to measure many lines of interest to much larger distances from the exciting star $\theta^{1}$ Ori $\mathrm{C}$.
\end{abstract}

Keywords. ISM: abundances, H II regions, individual (Orion Nebula)

\section{Synopsis}

Orion is the benchmark for studies of the interstellar medium, particularly for elemental abundances. Spitzer observations provide a unique perspective on the neon and sulfur abundances. The $\mathrm{Ne} / \mathrm{H}$ abundance ratio is especially well determined, with a preliminary value of $1.12 \times 10^{-4}$.

There are corresponding new ground-based spectra taken by Co-I Bob O'Dell. We find that the electron density vs. distance from $\theta^{1}$ Ori $\mathrm{C}$ for [S III] (Spitzer) and [S II] (CTIO) decreases in a very similar distribution from $\sim 1000$ to $\sim 100 \mathrm{~cm}^{-3}$. The optical data are used to estimate the electron temperature, optical extinction, and the $\mathrm{S}^{+}$fraction. From our preliminary results we estimate the $\mathrm{Ne} / \mathrm{S}$ abundance ratio by number by observing the dominant ionization states of $\mathrm{Ne}\left(\mathrm{Ne}^{+}, \mathrm{Ne}^{++}\right)$and $\mathrm{S}\left(\mathrm{S}^{++}, \mathrm{S}^{3+}\right)$ with Spitzer. The optical data are used to correct our Spitzer-derived Ne/S ratio for $\mathrm{S}^{+}$which is not observed with Spitzer. The median value (excluding all three outermost 'Veil' positions) adjusted for the optical $\mathrm{S}^{+} / \mathrm{S}^{++}$ratio is $\mathrm{Ne} / \mathrm{S}=14.5$.

A dramatic find is the presence of high-ionization $\mathrm{Ne}^{++}$all the way to the outer optical boundary $\sim 12$ arcmin from $\theta^{1}$ Ori C. This IR result is robust, whereas the optical evidence from observation of high-ionization (e.g. $\mathrm{O}^{++}$) at the outer optical boundary suffers uncertainty because of the scattering of emission from the much brighter inner Huygens region.

The Spitzer spectra are consistent with the Bright Bar being a high-density 'localized escarpment' in the larger Orion Nebula picture. Hard ionizing photons reach most solid angles well SE of the Bright Bar.

Our spectra are the deepest ever taken in these outer regions of Orion over the $10-37 \mu \mathrm{m}$ range. Tracking the changes in ionization structure via the line emission to larger distances provides much more leverage for understanding the far less studied outer regions. The outer Veil is likely a new $\mathrm{H}$ II region-PDR interface.

\section{Acknowledgements}

Support is from Spitzer Space Telescope Cycle 5 program 50082. This research is the combined effort of C.R. O’Dell, G. Ferland, J. Simpson, S. Colgan, \& I. McNabb. 